WellBeing International

WBI Studies Repository

$1-1995$

\title{
Responses to Quantity: Perceptual Versus Cognitive Mechanisms in Chimpanzees (Pan Troglodytes)
}

Sarah T. Boysen

The Ohio State University

Gary G. Berntson

Emory University

Follow this and additional works at: https://www.wellbeingintlstudiesrepository.org/acwp_asie

Part of the Animal Studies Commons, Comparative Psychology Commons, and the Other Animal

Sciences Commons

\section{Recommended Citation}

Boysen, S. T., \& Berntson, G. G. (1995). Responses to quantity: perceptual versus cognitive mechanisms in chimpanzees (Pan troglodytes). Journal of Experimental Psychology: Animal Behavior Processes, 21(1), 82.

This material is brought to you for free and open access by WellBeing International. It has been accepted for inclusion by an authorized administrator of the WBI Studies Repository. For more information, please contact wbisr-info@wellbeingintl.org.

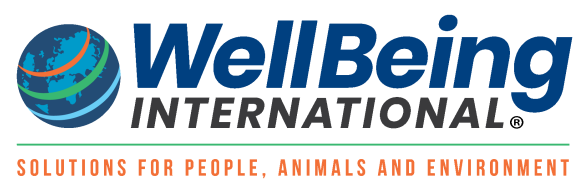




\title{
Responses to Quantity: Perceptual Versus Cognitive Mechanisms in Chimpanzees (Pan Troglodytes)
}

\author{
Sarah T. Boysen ${ }^{1,2}$ and Gary G. Berntson ${ }^{1,2}$ \\ ${ }^{1}$ Ohio State University \\ ${ }^{2}$ Emory University
}

\begin{abstract}
Two chimpanzees were trained to select among 2 different amounts of candy (1-6 items). The task was designed so that selection of either array by the active (selector) chimpanzee resulted in that array being given to the passive (observer) animal, with the remaining (nonselected) array going to the selector. Neither animal was able to select consistently the smaller array, which would reap the larger reward. Rather, both animals preferentially selected the larger array, thereby receiving the smaller number of reinforcers. When Arabic numerals were substituted for the food arrays, however, the selector animal evidenced more optimal performance, immediately selecting the smaller numeral and thus receiving the larger reward. These findings suggest that a basic predisposition to respond to the perceptualmotivational features of incentive stimuli can interfere with task performance and that this interference can be overridden when abstract symbols serve as choice stimuli.
\end{abstract}

Evaluation of numerical capabilities in animals in recent years has expanded the range of skills previously demonstrated in nonhuman species, provoked new questions regarding possible processing mechanisms that might subserve these capacities, and encouraged new directions for research (Boysen \& Capaldi, 1993; Thomas \& Lorden, 1993). Findings from our laboratory have indicated that the chimpanzee is an apt subject for the study of numerical competence and, with appropriate training, can demonstrate facility with counting, basic summation abilities, symbolically mediated summation, and an understanding of ordinality (Boysen, 1992, 1993; Boysen \& Berntson, 1989; Boysen, Berntson, Shreyer, \& Quigley, 1993). The establishment of such skills in chimpanzees is significantly labor intensive (Boysen, 1992) and requires years of gradually expanded training. Although this may appear at variance with the seemingly effortless acquisition of basic counting skills and number concepts in human children, an appraisal of the children's counting literature suggests quite a different picture. Counting in children entails a highly complex set of skills acquired gradually over years, facilitated by the emergence of verbal abilities and concomitant cognitive development (Fuson, 1988; Gallistel \& Gelman, 1992; Gelman \& Gallistel, 1978). 
Although the range of number skills acquired by chimpanzees may be more limited than possible in children, our animals have nevertheless been successful in each number-related task that has been undertaken, including those using numerical symbols (see Boysen, 1993, for an overview). An obvious question arises as to the nature of the symbolic representation evoked by Arabic numerals in animals with an extensive repertoire of numerical competence. The present study offers additional insights into this issue. Our initial goal was to explore the possible use of deception by chimpanzees who had previous training in counting and number comprehension (Boysen \& Berntson, 1989). Preliminary training, however, revealed that the animals were unable to perform optimally in a simple discrimination task that required the selection of the smaller of two arrays of food. This inability was striking in view of the complex numerical tasks that the animals had performed successfully. The current study explored this performance deficit and its alleviation by the use of numerical symbols. Results suggest that symbols may come to represent only selective attributes of their referents and that this selective representation may be fundamental to the ultimate adaptive utility of symbols.

\section{Method}

\section{Subjects}

Two female chimpanzees served as subjects for the study: Sheba, a 9-year-old, and Sarah, a 32-yearold. Both animals had extensive experience and training on a variety of cognitive and behavioral studies (e.g., Boysen, 1993; Boysen \& Berntson, 1989; Premack, 1986). Sheba had acquired significantly more elaborate skills with numbers than Sarah, who had joined the Comparative Cognition Project more recently (1987, from David Premack's laboratory). Sarah's counting repertoire was limited, with concurrent training underway on associations between arrays of 1 to 5 candies and their corresponding Arabic numerals. Sheba's counting repertoire was more extensive, including demonstrated facility with the numerals 0 to 8 , and she had participated in a study of ordinality and transitivity, which included a phase of training and testing with numerical stimuli (Boysen et al., 1993). Sheba also had significantly more experience in the numerical labeling of arrays of foods and objects, receptive number comprehension training, summation with objects or Arabic numerals, subtraction, and comprehension of fractions (Boysen, 1993; Boysen \& Berntson, 1989).

\section{Apparatus and Testing Context}

The apparatus consisted of a large $(1.3 \times 1.5 \mathrm{~m})$ plywood box, the ends of which served to define the animal's testing stations. Four stimulus and reward trays were located in the middle of the box. The 2 animals worked together during all phases of training and testing. Sarah was tested in her home cage, with the test apparatus placed up to the front of the cage. Sheba, who served as Sarah's partner, sat outside the cage on the other side of the test apparatus. Sheba was unrestrained during all testing but remained attentive and compliant to the task demands.

The experimenter was positioned at the side of the box and was responsible for loading stimulus trays and delivering rewards. After stimuli and reinforcers were placed in the trays, the experimenter moved behind a barrier out of view of the test animal. The animal then indicated a choice by pointing to the selected tray. The experimenter maintained a vantage (from behind the barrier) that permitted viewing of the stimulus trays and the animal's selection response. All training and testing sessions were videotaped, and all responses were concurrently recorded on data sheets by the experimenter.

\section{Procedure}

Initial training with candy arrays. For each trial, two arrays of candy (gumdrops or chocolate-covered peanuts) were placed in two of the four stimulus trays initially in ratios of 1:2, 1:4, or 1:6 candies. The two 
trays to be used on a given trial were selected randomly in an attempt to minimize position biases. One chimp was designated as the selector, and this animal was permitted to select one of the two trays (by pointing). In full sight of both animals, the experimenter then gave the contents of the selected tray to the other, observer chimpanzee, and the remaining candy array (in the nonselected dish) was given to the selector. Thus, it was to the selector's advantage to select the dish containing the smaller number of candies because she would then receive the larger array (in the nonselected dish).

Test sessions were conducted 5 days a week between 10 a.m. and 3 p.m. and consisted of 12 to 15 trials. Both animals were maintained on their normal food rations throughout the study (a variety of fresh fruits and vegetables given in feedings 3 to 5 times a day). Candy and other treats were used as reinforcers during testing on a variety of other tasks throughout the day and as rewards for compliant behavior (e.g., traded in exchange for soiled blankets). Thus, although candy remained a highly desirable item, it did not represent a scarce resource that was rarely available to the animals.

Sarah initially served as the selector animal, and on each trial, a single piece of candy was placed in one of the stimulus trays and two pieces were placed in the other (with position counterbalanced across trials). A total of 96 trials was given over eight sessions, with the contents of the selected dish going to the observer (Sheba), whereas the selector (Sarah) was given the candies in the remaining nonselected dish. The size of the larger array was then increased to four candies for eight additional sessions and then to six for eight additional sessions. A final eight sessions were completed with all three ratios presented on different trials.

At that point, the experimental roles of the two chimpanzees were reversed. Sheba now served as the selector animal, after considerable experience as the passive observer in the task. Eight sessions were given in which arrays of one and two, one and four, and one and six candies were randomly presented across trials for a total of 96 trials.

Testing with Arabic numerals. In view of the poor performance of the animals with candy arrays, the array stimuli were replaced with Arabic numerals. The testing context was identical to that described previously except that the candy arrays no longer served as test stimuli. Instead, Arabic numerals 1, 2, 4, and 6 (black numerals on a white background affixed to $7.5 \times 12-\mathrm{cm}$ plastic placards) were substituted for the arrays of candies. Because Sheba was more accomplished with numerical symbols, she continued in her role as selector animal. She was now required to choose between numerals 1 versus 2,1 versus 4 , or 1 versus 6 placed in the two otherwise empty stimulus trays. In accord with the general contingency used previously, the numeral selected by Sheba resulted in a corresponding number of candies being given to the observer (Sarah). Sheba then received the number of candies that corresponded to the remaining nonselected numeral from the other stimulus tray. After three sessions using numerical stimuli only, six additional test sessions were completed in an ABBA design, with A sessions using Arabic numerals as test stimuli and B sessions using candy arrays.

Testing with novel array combinations. Results from this testing indicated that Sheba was able to respond optimally with numerical stimuli but not with candy arrays. To evaluate whether Sheba had simply learned to select the numeral 1 when numerals were used as stimuli and to examine the effects of the numerical disparity between the choice stimuli, an additional series of test sessions were completed. The same general procedures were used, with the exception that all possible array combinations of numerals 1 to 6 and candy arrays composed of one to six items were used as test stimuli. A total of eight sessions (15 trials each) were given, four with Arabic numerals and four with candy arrays (in counterbalanced ABBA order across sessions). Within each session, trials consisted of random combinations of stimuli (one to six, excluding equal arrays). Sheba continued to serve in the selector role. 


\section{Results}

Initial Training With Food Arrays

In striking contrast to the facility with which these highly trained chimpanzee subjects typically acquired most tasks, neither animal was able to reliably select the smaller of two candy arrays to receive a larger nonselected array. Sarah initially served as the selector animal, with Sheba as the observer (and the recipient of the selected array). Sarah's performance over the first three eight-session (96-trial) blocks with arrays of one versus two, one versus four, and one versus six candies, respectively, hovered around chance $(57 \%, 57 \%$, and $64 \%$, respectively). As illustrated in Table 1, Sarah also failed to improve in a final eight-session (96-trial) block with all array ratios represented across trials. Her overall performance did not differ from chance for any of the array ratios (one vs. two, 58\%; one vs. four, 44\%; one vs. six, 44\%). Thus, after nearly 400 training trials, extending over 32 sessions, Sarah was unable to acquire an optimal response strategy of selecting the smaller of two candy arrays to obtain the larger nonselected array.

When social roles on the task were reversed, Sheba also exhibited great difficulty in acquiring an optimal response strategy for reaping the greater number of reinforcements. Her overall performance on eight mixed-ratio sessions (see Table 1) was no better than Sarah's. In fact, Sheba's performance was significantly below chance at $34 \%$ correct, $X^{2}(1, N=2)=14.8, p<.001$. Moreover, her performance became less optimal at higher reward ratios when Sheba stood to benefit the most (performance on 1:2 ratio, 44\%; 1:4, 22\%; 1:6, 28\%; $p<.05)$.

Table 1. Performance of Chimpanzees When Maximal Reward Depends on the Selection of the Smaller of Two Candy Arrays

\begin{tabular}{|cccccc|}
\hline & \multicolumn{2}{c}{ Sarah } & & \multicolumn{2}{c|}{ Sheba } \\
\cline { 2 - 3 } \cline { 5 - 6 } Session & Correct trials $/$ total trials & $\%$ & & Correct trials / total trials & $\%$ \\
\hline 1 & $6 / 12$ & 50 & & $2 / 12$ & 17 \\
\hline 2 & $9 / 12$ & 55 & $2 / 12$ & 17 \\
\hline 3 & $7 / 12$ & 58 & $5 / 12$ & 17 \\
\hline 4 & $5 / 12$ & 42 & $6 / 12$ & 52 \\
\hline 5 & $4 / 12$ & 33 & $6 / 12$ & 50 \\
\hline 6 & $4 / 12$ & 33 & $4 / 12$ & 53 \\
\hline 7 & $4 / 12$ & 33 & $6 / 12$ & 50 \\
\hline 8 & $8 / 12$ & 67 & $33 / 96$ & 34 \\
\hline$M$ & $47 / 96$ & 49 & &
\end{tabular}

\section{Performance With Symbols as Stimuli}

Given the poor performance with food arrays and Sheba's established proficiency in the use of numerical symbols, Arabic numerals were substituted for the candy arrays. Sheba continued in the role of selector, and arrays of 1 versus 2, 1 versus 4, and 1 versus 6 were tested. When Arabic numerals were introduced, Sheba reliably selected the smaller of the two numerals, thereby receiving the larger amount of reinforcement $75 \%$ of the time (three sessions, 36 trials, $75 \%$ correct), $x^{2}(1, N=2)=9.0, p<.01$. Moreover, Sheba responded correctly on the very first trial, and her performance remained stable over the three sessions $(67 \%, 75 \%$, and $83 \%$, respectively). 
Candies and numerals were then alternated as stimuli over the next six sessions (ABBA order). Sheba's mean performance across the three sessions with candies was 17\% (2 correct of 12 trials in each session), whereas her mean performance over the three sessions with the symbolic stimuli was $72 \%$ ( 9 , 8 , and 9 correct of 12 trials, respectively). These differences in performance were stable and highly significant, $X^{2}(1, N=2)=7.1, p<.01$. Sheba could respond adaptively when Arabic numerals served as stimuli, but with candy arrays she continued to select the larger of the two quantities, and the observer animal typically received the greater number of reinforcers.

\section{Performance With Novel Array Ratios}

To evaluate whether Sheba had simply learned to select the numeral 1 when numerals were used as stimuli and to examine the effect of array size disparity, an additional series of test sessions were completed in which all possible combinations of array sizes (from 1 to 6) were presented in an ABBA design. As shown in Figure 1, Sheba reliably selected the smaller array (and thus received the larger reward) when given a choice between novel combinations of Arabic numerals but continued to choose the larger array (and thus received the smaller reward) when food arrays were used as stimuli.

During sessions with Arabic numerals, Sheba's performance was significantly above chance (total correct: 42 of 60 trials, or $70 \%), x^{2}(1, N=2)=9.6, p=.001$, but with food arrays as stimuli, her performance fell significantly below chance (total correct: 13 of 60 trials, or $22 \%), x^{2}(1, N=2)=19.6, p<$ .001 . Moreover, Sheba's performance was greatly affected by the arithmetic disparity between the choice stimuli. As is apparent in Figure 1, with minimal disparity among the choice stimuli, performance with candy arrays and Arabic numerals differed only slightly, both being around 50\%. However, when candy arrays were used as choice stimuli, Sheba's performance deteriorated as the difference between the numbers of candies in the two arrays increased. In contrast, when Arabic numerals were used, her performance improved as the numerical disparity between the two numbers increased (see Figure 1). That is, increasing differences in the size of candy arrays led to progressively less adaptive responding, whereas increasing disparities between numerical symbols resulted in more optimal performance.

\section{Discussion}

The present results pose a significant challenge for understanding why a seemingly simple discrimination task, with rather trivial demands, proved so difficult for two highly trained chimpanzees. The animals were unable to select the smaller of two food arrays even when that response would yield a larger reward. The fact that the animals exhibited considerable behavioral distress (e.g., vocalizations and striking the apparatus) over the inequitable distribution of candy indicates that the response consequences were well appreciated by the subjects. Moreover, performance of one of the animals (Sheba) was significantly below chance, suggesting the operation of a potent response determinant that opposed the task-defined contingency.

Importantly, the disposition to respond to the larger food arrays did not appear to have prevented learning of the rules and contingencies of the task. Although Sheba was not able to reliably select the smaller of two food arrays, she displayed good performance when numerical symbols were substituted for the food arrays. Moreover, her performance improved immediately on introduction of the symbolic stimuli without the requirement for additional training. These findings suggests that Sheba had, in fact, acquired an understanding of the food-division rules that had been enforced since the start of the study. Rather, it appears that the animals were unable to inhibit a competing evaluative disposition to select the larger of the arrays when food items were used as stimuli. In this regard, the interference effect likely reflected the evaluative gradient associated with differences in the intrinsic incentive properties of the task stimuli. This was further supported by the fact that, with increasing numerical disparity among the choice stimuli, 
performance with food arrays deteriorated but performance with Arabic numerals improved. Thus, it appears that two dispositional gradients are operative in this task: one related to the experimentally imposed reward contingency, and another from the direct incentive properties of the perceptual arrays. These dispositions (a) arise from distinct antecedents, (b) manifest differentially in performance, and (c) interact. The associative gradient arising from the reinforcement contingency may be masked or overridden by the competing nonassociative disposition related to the intrinsic incentive properties of the candy array stimuli.

Figure 1. Performance of a chimpanzee on a paired-choice task in which selection of the smaller quantity yields the larger reward. Left panel: Performance over sessions as the choice stimuli were switched between Arabic numerals and food arrays. A correct response is considered the selection of the smallest quantity, which yields the largest reward. Right panel: Performance with numerals and food arrays as a function of the arithmetic disparity between the two choice stimuli of a given trial. (Prob. $=$ probability)
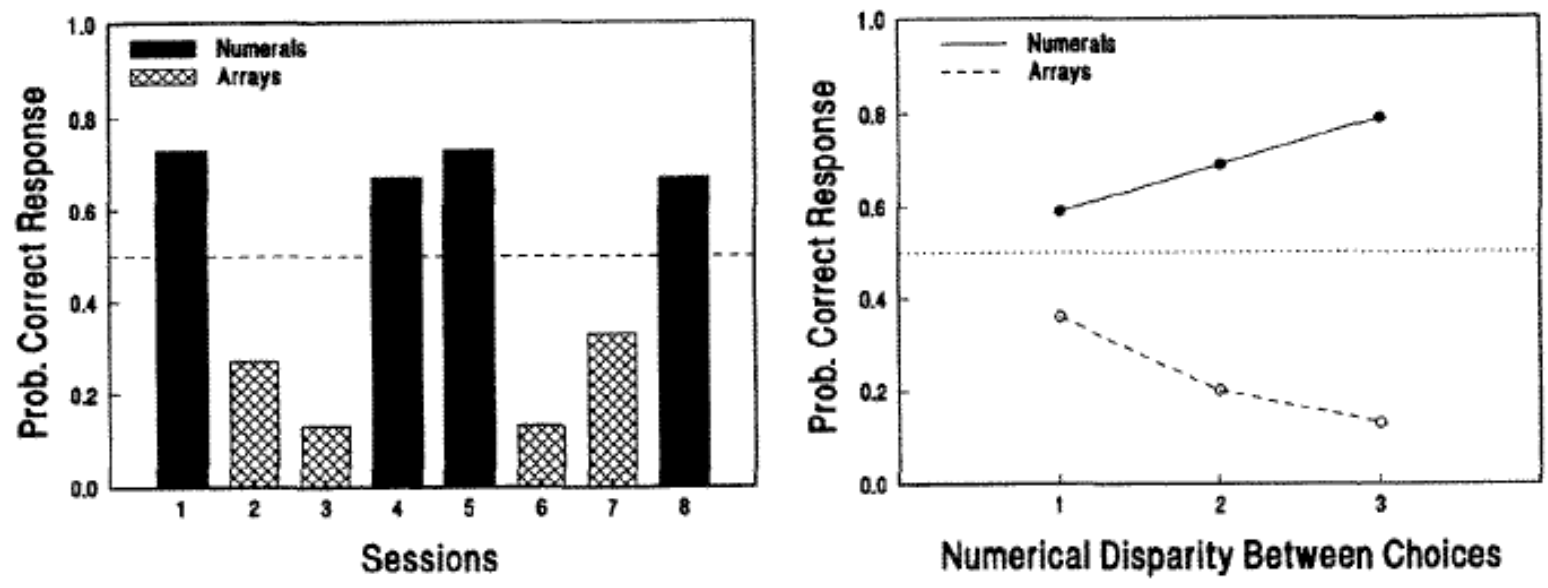

The poor performance with candy arrays observed in the present study may reflect a simple but generally adaptive perceptual strategy that would likely be appropriate under natural foraging conditions, a strategy that would permit a chimpanzee to respond to a larger, more concentrated food source with minimal higher level cognitive processing. The use of symbols as representations for quantities of foods, however, may free the animals from the dispositional imperative invoked by the direct perceptual features of the food arrays, thus permitting more optimal responding to maximum reward.

There is precedence for similar inherent tendencies of chimpanzees, when faced with differing amounts of food, to consistently select the larger amount (e.g., Menzel, 1960). Moreover, the type of preparedness observed in these experiments may also explain the findings reported by Rumbaugh, Savage-Rumbaugh, and Hegel (1987). In this study, chimpanzees were permitted to select from two pairs of candy arrays and generally chose the larger aggregate. The results of the current study raise the possibility that the Rumbaugh et al. (1987) data were inevitable, reflecting not a counting process or summation but a perceptual-dispositional imperative. In view of the present results, had these investigators attempted to demonstrate the reverse (selection of the smaller of two separate arrays), the chimps would likely have failed.

We have observed an apparently similar imperative phenomenon during the introduction of a numbercomprehension task that suggests some generality of the present results. This task required that the animals select a food array equal to an Arabic numeral (Boysen \& Berntson, 1989). In this task, the 
chimps also had great difficulty inhibiting the selection of the larger of two arrays instead of matching the correct food array with a displayed Arabic numeral. With respect to the food-sharing task described here, we may have been attempting to train the animals on a discrimination for which they already had some highly prepared "privileged relationship" with quantities (Gallistel, Brown, Carey, Gelman, \& Keil, 1991, p. 5).

In the present study, the introduction of representative symbols provided the animal with an opportunity to override this perceptual-motivational predisposition. This permitted the use of symbolic information, which significantly enhanced performance. In this regard, the present results differ notably from the earlier report of Breland and Breland (1961) and from numerous examples in the autoshaping or stimulusequivalence literatures in which a conditioned stimulus or token symbol may come to evoke an evaluative reaction highly reminiscent of the response to the unconditioned stimulus. Although this is a complex issue that cannot adequately be dealt with here, one likely contribution to the outcome of the present study is apparent. Sheba had been extensively trained with numerical symbols in many experimental contexts, extending over many years. Throughout this training, the stimulus referents differed considerably from study to study and from even trial to trial. Moreover, in many instances, nonincentive stimuli (e.g., junk objects) were used. Indeed, as an integral aspect of this training, numerical symbols were never consistently associated with any particular stimulus. Rather, the uniform focus of this training was on the relationship between Arabic numerals and the number of elements in stimulus array independent of their unique features or incentive values.

In summary, it appears that the intrinsic incentive properties of the candy array stimuli invoked an evaluative disposition that interfered with optimal performance. In the present task, however, although this dispositional bias powerfully affected behavioral choice, it did not appear to preclude acquisition of the rule structure of the task. By liberating the animal from the dictates of this potent evaluative disposition, symbolic stimuli permitted the expression of the associative knowledge of this rule structure. Indeed, part of the power of symbolic representations such as language in humans may relate to the ability of these representations to capture selected aspects or relationships among their real-world referents while liberating the organism from other aspects.

\section{References}

Boysen, S. T. (1992). Counting as the chimpanzee views it. In W. K. Honig \& J. G. Fetterman (Eds.), Cognitive aspects of stimulus control (pp. 367-383). Hillsdale, NJ: Erlbaum.

Boysen, S. T. (1993). Counting in chimpanzees: Nonhuman principles and emergent properties of number. In S. T. Boysen \& E. J. Capaldi (Eds.), The development of numerical competence: Animal and human models (pp. 39-59). Hillsdale, NJ: Erlbaum.

Boysen, S. T., \& Berntson, G. G. (1989). Numerical competence in a chimpanzee (Pan troglodytes). Journal of Comparative Psychology, 103, 23-31.

Boysen, S. T., Berntson, G. G., Shreyer, T. A., \& Quigley, K. S. (1993). Processing of ordinality and transitivity by chimpanzees (Pan troglodytes). Journal of Comparative Psychology, 107, 208-215.

Boysen, S. T. \& Capaldi, E. J. (1993). The development of numerical competence: Animal and human models. Hillsdale, NJ: Erlbaum.

Breland, K., \& Breland, M. (1961). The misbehavior of organisms. American Psychologist, 16, 682-684.

Fuson, K. C. (1988). Children's counting and concepts of number. New York: Springer-Verlag.

Gallistel, C. R., Brown, A. L., Carey, S., Gelman, R., \& Keil, F. C. (1991). Lessons from animal learning for the study of cognitive development. InS. Carey \& R. Gelman (Eds.), The epigenesis of mind: Essays on biology and cognition (pp. 3-36). Hillsdale, NJ: Erlbaum.

Gallistel, C. R., \& Gelman, R. (1992). Preverbal and verbal counting and computation. Cognition, 44, 4374. 
Gelman, C. R., \& Gallistel, C. R. (1978). The child's understanding of number. Cambridge, MA: Harvard University Press.

Menzel, E. (1960). Selection of food by size in the chimpanzee and comparison with human judgments. Science, 131, 1527-1528.

Premack, D. (1986). Gavagai. London: Cambridge University Press.

Rumbaugh, D. M., Savage-Rumbaugh, E. S., \& Hegel, M. (1987). Summation in the chimpanzee (Pan troglodytes). Journal of Experimental Psychology: Animal Behavior Processes, 13, 107-115.

Thomas, R. K., \& Lorden, R. B. (1993). Numerical competence in animals: A conservative view. In S. T. Boysen \& E. J. Capaldi (Eds.), The development of numerical competence: Animal and human models (pp. 127-147). Hillsdale, NJ: Erlbaum. 